\title{
Synthesis of 3,4,5-trisubstituted indoles via iterative directed lithiation of 1-(triisopropylsilyl)gramines
}

\author{
Tsutomu Fukuda, Hiroko Akashima, and Masatomo Iwao* \\ Department of Applied Chemistry, Faculty of Engineering, Nagasaki University, 1-14 \\ Bunkyo-machi, Nagasaki 852-8521, Japan
}

\begin{abstract}
Directed lithiation of 1-(triisopropylsilyl)gramines 1 with tert-butyllithium followed by reaction with trimethylsilylmethyl azide produced 4-amino-1-(triisopropylsilyl)gramines 7. The $N$-tert-butoxycarbonyl derivatives 8 were lithiated selectively at C-5 with tert-butyllithium and the lithiated species were reacted with a variety of electrophiles to give 5-functionalized compounds, 9 and $\mathbf{1 0}$. A facile method to produce 3,4,5-trisubstituted indoles from readily available gramine derivatives is thereby established.
\end{abstract}

\section{Introduction}

Functionalization at the 1-, 2-, and 3-positions of the indole ring can be effected easily by conventional methods. ${ }^{1}$ On the other hand, regioselective substitution at the benzenoid portion is rather problematic. Consequently, the development of procedures to achieve this objective has been a challenge for synthetic chemists for many years. ${ }^{2}$ 
In 1993, we reported a facile method to produce 4-substituted indoles via directed lithiation of 1-(triisopropylsilyl)gramine (1a) (Scheme 1). ${ }^{2 \mathrm{~g}}$ The selective lithiation at the 4-position is achieved by both the ortho-directing effect of the $N, N$-dimethylaminomethyl group and the steric shielding of the proton at $\mathrm{C}-2$ by a bulky $\mathrm{N}$-triisopropylsilyl group. The synthetic utility of this reaction has been expanded by development of a procedure for further elaboration at the C-3 side chain via the fluoride-induced elimination-addition reaction of 1-(triisopropylsilyl)gramine methiodides (Scheme 2). ${ }^{3} \quad$ The combination of these reactions allows short-step synthesis of a wide range of 3,4-disubstituted indoles 3 , including biologically significant natural products and their analogues, such as clavicipitic acids, ${ }^{4}$ pyrroloiminoquinone marine alkaloids, ${ }^{5}$ indolactam- and teleocidin-class PKC regulators, ${ }^{6}$ 4-fluoroserotonine and -melatonine, ${ }^{7}$ and so on. ${ }^{8}$ Halonium-induced retro-Mannich reaction, recently reported by Snieckus, allows the ring functionalization at C-3 of the gramines (Scheme 2). ${ }^{9}$

\section{Scheme 1}

\section{Scheme 2}

Iterative directed lithiation proposed by Snieckus is a potentially valuable method to produce multisubstituted aromatics in short steps. ${ }^{10,21}$ The process is a series of lithiation-electrophilic substitution in which a newly created directing group promotes the next lithiation. We intended to apply this methodology for the synthesis of 3,4,5-trisubstituted indoles starting from 1-(triisopropylsilyl)gramine (1a), because no general synthetic approach to such indoles has been reported. The concept is shown in Scheme 3. The initial C-4 lithiation of 1a followed by quenching with an appropriate 
electrophile produces 4 -substituted gramine $\mathbf{5}$ having a directing group (DG) at C-4, which can promote the next lithiation at C-5 to give a variety of 3,4,5-trisubstituted indoles 6 .

\section{Scheme 3}

\section{Results and discussion}

In the synthetic transformation described above, the choice of the directing group at C-4 may be most important. From a practical point of view, we selected tert-butoxycarbonylamino (Boc-NH) group as a director, because 1) good directing ability of Boc-NH has been established in the ortho-lithiation of aniline derivatives, ${ }^{11}$ ) 4-amino-1-(triisopropylsilyl)gramine is readily available in high yield via directed lithiation of $1,{ }^{2 \mathrm{~g}, 6} 3$ ) the amino group at $\mathrm{C}-4$ of the indoles could be readily transformed to a variety of functionalities via diazonium salt displacement reactions, ${ }^{12}$ and finally 4 ) some biologically significant natural products comprise a 4-aminoindole substructure in their molecular framework. ${ }^{5,6}$

The synthesis of 4-(N-tert-butoxycarbonyl)amino-1-(triisopropylsilyl)gramines $\mathbf{8}$ is shown in Scheme 4. Directed lithiation of $\mathbf{1}$ under the established conditions (tert-butyllithium, diethyl ether, $-78{ }^{\circ} \mathrm{C}, 15 \mathrm{~min}$, then $\left.0{ }^{\circ} \mathrm{C}, 1.5 \mathrm{~h}\right)^{2 \mathrm{~g}}$ followed by reaction with trimethylsilylmethylazide ${ }^{13}$ produced 4 -aminogramines $7 \mathbf{a}$ and $\mathbf{7 b}$ in 79 and $86 \%$ yields, respectively. Treatment of 7 with di-tert-butyl dicarbonate in refluxing THF gave the corresponding $N$-tert-butoxycarbonyl derivatives $\mathbf{8}$. 


\section{Scheme 4}

Ortho-lithiation of $N$-(tert-butoxycarbonyl)aniline was achieved for the first time by Muchowski in $1980 .{ }^{11 a}$ The compound was lithiated with tert-butyllithium in THF at $-20{ }^{\circ} \mathrm{C}$. In 1992, Stanetty reexamined this reaction precisely and discovered that utilization of diethyl ether instead of THF as a solvent is essential for good and reproducible results. ${ }^{11 \mathrm{~d}}$ Thus, we employed the conditions similar to Stannety's for the lithiation of $\mathbf{8}$. After some optimization studies using iodomethane as an electrophile, we found that the selective C-5 lithiation can be effected most satisfactorily by treatment of $8 \mathbf{a}$ in diethyl ether with 3.0 equiv. of tert-butyllithium at $-78{ }^{\circ} \mathrm{C}$ for $15 \mathrm{~min}$ and then at $0{ }^{\circ} \mathrm{C}$ for $1 \mathrm{~h}$. The lithiated species was reacted with a range of electrophiles at $0{ }^{\circ} \mathrm{C}$ for $1 \mathrm{~h}$ to give 5 -substituted compounds 9 a-g in good isolated yields (Table 1 , entries 1-7). Utilization of a slight excess of electrophile (1.5 equiv to the substrate) is enough to trap the lithiated species. This means excess tert-butyllithium was decomposed by the reaction with the solvent under the lithiation conditions. ${ }^{11}$ A substrate 8b having a methoxy group at C-6 was also lithiated at C-5 selectively under similar conditions. However, the lithiated species was found to be somewhat unstable under the lithiation conditions and, after quenching with electrophiles, the C-5 substituted products 10a-g were isolated in moderate yields (Table, entries 8-14). ${ }^{14}$

Table

\section{Conclusion}

We have developed a general synthetic route to 3,4,5-trisubstituted indoles from readily 
available gramine derivatives via an iterative directed lithiation strategy. In view of the facile substitution at C-3 (side chain or ring) of the gramines and the C-4 functionalization of 4-aminoindoles via diazonium salts, the present procedure may open the way to diverse 3,4,5-trisubstituted indoles, which are not readily available by conventional synthetic methodology.

\section{Experimental}

\subsection{General.}

Melting points were determined with a Yanagimoto micro melting points apparatus and are uncorrected. IR spectra were obtained with a Perkin-Elmer System 2000 instrument. NMR spectra were recorded on a JEOL JNM-AL400 instrument (400 $\mathrm{MHz}$ for ${ }^{1} \mathrm{H}$ and $100 \mathrm{MHz}$ for ${ }^{13} \mathrm{C}$ ) using tetramethylsilane as an internal standard. Column chromatography was conducted on Aluminum oxide 90 standardized (Merck KGaA), or Silica Gel 60N, 63-210 um (Kanto Chemical Co., Inc.). tert-Butyllithium was purchased from Aldrich Chemical Co., Inc. and used after titration with 2,5-dimethoxybenzyl alcohol. Diethyl ether and THF were dried over Na-benzophenone ketyl under Ar and distilled immediately before use. 1-(Triisopropylsilyl)gramine (1a) ${ }^{4 a}$ 6-methoxy-1-(triisopropylsilyl)gramine (1b), ${ }^{5 a}$ and trimethylsilylmethyl azide ${ }^{13}$ were prepared according to the reported procedures.

\subsection{Procedure for the synthesis of 4-amino-1-(triisopropylsilyl)gramines 7 .}

Under an argon atmosphere, a pentane solution of tert-butyllithium (12 mmol) was 
added dropwise to a solution of $\mathbf{1}(10 \mathrm{mmol})$ in diethyl ether $(50 \mathrm{~mL})$ at $-78{ }^{\circ} \mathrm{C}$. After being stirred for $15 \mathrm{~min}$, the reaction mixture was allowed to warm to $0{ }^{\circ} \mathrm{C}$ and stirred for an additional $1.5 \mathrm{~h}$ at the same temperature. The reaction mixture was cooled to $-78{ }^{\circ} \mathrm{C}$, and a solution of trimethylsilylmethyl azide $(1.94 \mathrm{~g}, 15 \mathrm{mmol})$ in diethyl ether (3 mL) was added dropwise. After being stirred for $1 \mathrm{~h}$, the reaction mixture was allowed to warm to room temperature and quenched with saturated aqueous $\mathrm{NH}_{4} \mathrm{Cl}$. The products were extracted with diethyl ether and the extract was washed successively with water and brine, dried over $\mathrm{Na}_{2} \mathrm{SO}_{4}$, and evaporated under reduced pressure. The residue was purified by column chromatography over Aluminum oxide 90 standardized (hexane-ethyl acetate=10:1) to give 7 .

4.2.1. 4-Amino-1-(triisopropylsilyl)gramine (7a). According to the procedure described above, $1 \mathbf{a}(3.31 \mathrm{~g}, 10 \mathrm{mmol})$ was reacted to give $\mathbf{7 a}$ as pale yellow solid (2.75 g, 79\%). $\quad$ Mp 97-97.5 ${ }^{\circ} \mathrm{C}$ (pentane); IR (KBr): 3415, 3283, 3165, 3052, 2942, 2866, $2824,1619,1585,1560,1491,1459,1438,1375,1315,1284,1245,1130,1073,1035$, 1017, 1001, 883, 724, 693, 658, 574, $512 \mathrm{~cm}^{-1} ;{ }^{1} \mathrm{H}$ NMR (400 MHz, $\left.\mathrm{CDCl}_{3}\right): \delta 1.13$ (d, $J=7.6 \mathrm{~Hz}, 18 \mathrm{H}), 1.59-1.71(\mathrm{~m}, 3 \mathrm{H}), 2.25(\mathrm{~s}, 6 \mathrm{H}), 3.54(\mathrm{~s}, 2 \mathrm{H}), 5.43($ br s, $2 \mathrm{H}), 6.31(\mathrm{~d}$, $J=7.4 \mathrm{~Hz}, 1 \mathrm{H}), 6.82(\mathrm{~d}, J=8.4 \mathrm{~Hz}, 1 \mathrm{H}), 6.87-6.92(\mathrm{~m}, 2 \mathrm{H}) ;{ }^{13} \mathrm{C}$ NMR $(100 \mathrm{MHz}$, $\left.\mathrm{CDCl}_{3}\right): \delta 12.80,18.15,44.55,56.57,104.01,104.32,115.89,119.61,122.59,128.11$, 142.38, 143.32. Anal. Calcd for $\mathrm{C}_{20} \mathrm{H}_{35} \mathrm{~N}_{3} \mathrm{Si}: \mathrm{C}, 69.51 ; \mathrm{H}, 10.21 ; \mathrm{N}, 12.16$. Found: $\mathrm{C}$, 69.48; H, 10.38; N, 12.04.

4.2.2. 4-Amino-6-methoxy-1-(triisopropylsilyl)gramine (7b). According to the procedure described above, $\mathbf{1 b}(7.21 \mathrm{~g}, 20 \mathrm{mmol})$ was reacted to give $\mathbf{7 b}$ as pale brown solid $(6.47 \mathrm{~g}, 86 \%)$. This compound was somewhat unstable and used for the next

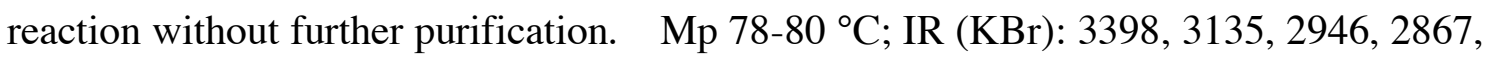


$2821,1616,1589,1561,1464,1200,1161,1128,1012,882,692,652,515 \mathrm{~cm}^{-1} ;{ }^{1} \mathrm{H}$ NMR (400 MHz, $\left.\mathrm{CDCl}_{3}\right): \delta 1.13(\mathrm{~d}, J=7.3 \mathrm{~Hz}, 18 \mathrm{H}), 1.57-1.67$ (m, 3H), $2.24(\mathrm{~s}, 6 \mathrm{H})$, 3.50 (s, 2H), 3.77 (s, 3H), 5.47 (br s, 2H), 6.01 (d, $J=2.0 \mathrm{~Hz}, 1 \mathrm{H}), 6.36$ (d, $J=2.0 \mathrm{~Hz}$, $1 \mathrm{H}), 6.78(\mathrm{~s}, 1 \mathrm{H}) ;{ }^{13} \mathrm{C}$ NMR $\left(100 \mathrm{MHz}, \mathrm{CDCl}_{3}\right): \delta 12.78,18.18,44.52,55.42,56.55$, 88.53, 93.52, 114.32, 115.88, 126.86, 142.63, 143.79, 157.02. Anal. Calcd for $\mathrm{C}_{21} \mathrm{H}_{37} \mathrm{~N}_{3} \mathrm{OSi}$ C, $67.15 ; \mathrm{H}, 9.93$; N, 11.19. Found: C, 67.27; H, 10.27; N, 11.11.

\subsection{Procedure for the of}

\section{4-(N-tert-butoxycarbonyl)amino-1-(triisopropylsilyl)gramines 8.}

Di-tert-butyl dicarbonate $(1.40 \mathrm{~g}, 6.4 \mathrm{mmol})$ was added as a neat liquid to a solution of $7(6.1 \mathrm{mmol})$ in THF $(30 \mathrm{~mL})$ at room temperature and the solution was refluxed for $2 \mathrm{~h}$. The reaction mixture was then cooled to room temperature, and evaporated under reduced pressure. The residue was purified by column chromatography over Silica Gel $60 \mathrm{~N}$ (hexane-ethyl acetate $=10: 1$ ) to give $\mathbf{8}$.

\subsection{1. $\quad$ 4-(N-tert-Butoxycarbonyl)amino-1-(triisopropylsilyl)gramine}

(8a).

According to the procedure described above, 7a $(2.11 \mathrm{~g}, 6.1 \mathrm{mmol})$ was reacted to give 8a as colorless solid (2.46 g, 91\%). Recrystallization from hexane gave colorless prisms. Mp 102.5-103.5 ${ }^{\circ} \mathrm{C}$; IR (KBr): 3124, 2946, 2869, 2825, 2779, 1715, 1624, $1583,1557,1488,1458,1419,1288,1245,1158,1015,1001,882,735,663,578,513$ $\mathrm{cm}^{-1} ;{ }^{1} \mathrm{H}$ NMR $\left(400 \mathrm{MHz}, \mathrm{CDCl}_{3}\right): \delta 1.12(\mathrm{~d}, J=7.5 \mathrm{~Hz}, 18 \mathrm{H}), 1.53(\mathrm{~s}, 9 \mathrm{H}), 1.60-1.72$ $(\mathrm{m}, 3 \mathrm{H}), 2.31(\mathrm{~s}, 6 \mathrm{H}), 3.54(\mathrm{~s}, 2 \mathrm{H}), 6.96(\mathrm{~s}, 1 \mathrm{H}), 7.05-7.11(\mathrm{~m}, 2 \mathrm{H}), 7.69$ (br s, 1H), 11.61 (br s, $1 \mathrm{H}) ;{ }^{13} \mathrm{C}$ NMR $\left(100 \mathrm{MHz}, \mathrm{CDCl}_{3}\right): \delta 12.78,18.09,28.55,43.97,55.95$, $78.44,108.25,109.58,114.83,121.60,122.43,128.91,133.25,142.83,154.04$. Anal. Calcd for $\mathrm{C}_{25} \mathrm{H}_{43} \mathrm{~N}_{3} \mathrm{O}_{2} \mathrm{Si}$ : C, 67.37; H, 9.72; N, 9.43. Found: C, 67.02; H, 9.76; N, 9.39. 


\subsubsection{4-( $N$-tert-Butoxycarbonyl)amino-6-methoxy-1-(triisopropylsilyl)gramine (8b).}

According to the procedure described above, $7 \mathbf{b}(5.63 \mathrm{~g}, 15 \mathrm{mmol})$ was reacted to give 8b as colorless solid (5.70 g, 80\%). Recrystallization from hexane gave colorless prisms. $\quad$ Mp 108.5-109.5 ${ }^{\circ} \mathrm{C}$; IR (KBr): 3112, 2947, 2868, 1712, 1639, 1583, 1466, $1412,1279,1200,1163,1133,1016,884,839,683,655,593,586,517 \mathrm{~cm}^{-1} ;{ }^{1} \mathrm{H}$ NMR $\left(400 \mathrm{MHz}, \mathrm{CDCl}_{3}\right): \delta 1.12(\mathrm{~d}, J=7.6 \mathrm{~Hz}, 18 \mathrm{H}), 1.53(\mathrm{~s}, 9 \mathrm{H}), 1.60-1.70(\mathrm{~m}, 3 \mathrm{H}), 2.31(\mathrm{~s}$, 6H), $3.50(\mathrm{~s}, 2 \mathrm{H}), 3.84(\mathrm{~s}, 3 \mathrm{H}), 6.64(\mathrm{~d}, J=2.1 \mathrm{~Hz}, 1 \mathrm{H}), 6.85(\mathrm{~s}, 1 \mathrm{H}), 7.53$ (br s, 1H), 11.78 (br s, $1 \mathrm{H}) ;{ }^{13} \mathrm{C}$ NMR $\left(100 \mathrm{MHz}, \mathrm{CDCl}_{3}\right): \delta$ 12.76, 18.12, 28.54, 43.91, 55.88, $55.90,78.52$, 93.83, 97.84, 114.69, 116.11, 127.70, 133.57, 143.23, 153.91, 156.60 . Anal. Calcd for $\mathrm{C}_{26} \mathrm{H}_{45} \mathrm{~N}_{3} \mathrm{O}_{3} \mathrm{Si}: \mathrm{C}, 65.64 ; \mathrm{H}, 9.53 ; \mathrm{N}, 8.83$. Found: C, 65.59; H, 9.59; N, 8.84.

\subsection{Selective C-5 lithiation-electrophilic substitution of 4-(N-tert-butoxycarbonyl)amino-1-(triisopropylsilyl)gramines $8 . \quad$ General procedure}

Under an argon atmosphere, a pentane solution of tert-butyllithium (1.4 mmol) was added dropwise to a solution of $8(0.45 \mathrm{mmol})$ in diethyl ether $(4.5 \mathrm{~mL})$ at $-78{ }^{\circ} \mathrm{C}$. After being stirred for $15 \mathrm{~min}$, the reaction mixture was allowed to warm to $0{ }^{\circ} \mathrm{C}$ and stirred for an additional $1 \mathrm{~h}$ at the same temperature. A solution of an appropriate electrophile $(0.68 \mathrm{mmol})$ in diethyl ether $(3 \mathrm{~mL})$ was added and the solution was stirred for an additional $1 \mathrm{~h}$ at $0{ }^{\circ} \mathrm{C}$. The reaction mixture was quenched with saturated aqueous $\mathrm{NH}_{4} \mathrm{Cl}$ at the same temperature and allowed to warm to room temperature. The products were extracted with diethyl ether and the extract was washed successively with water and brine, dried over $\mathrm{Na}_{2} \mathrm{SO}_{4}$, and evaporated under reduced pressure. The 
residue was purified by column chromatography over Silica gel $60 \mathrm{~N}$ using the following eluents: hexane-ethyl acetate $=5: 1$ for $9 \mathbf{a}$, hexane-ethyl acetate $=3: 1$ for $\mathbf{9 b}, \mathbf{9 c}$, and 9d, hexane-ethyl acetate=5:1 3:1 for 9e, hexane-ethyl acetate=1:1 for 9f, hexane-ethyl acetate=1:2 for $9 \mathrm{~g}$, hexane-ethyl acetate=2:1 for $\mathbf{1 0 a}, \mathbf{1 0 b}, \mathbf{1 0 c}, \mathbf{1 0 e}$, and 10f, hexane-ethyl acetate $=2: 1 \sim 1: 1$ for $\mathbf{1 0 d}$, ethyl acetate for $10 \mathrm{~g}$. The results are shown in Table.

\subsubsection{4-(N-tert-Butoxycarbonyl)amino-5-methyl-1-(triisopropylsilyl)gramine (9a).}

According to the general procedure, 8a $(201 \mathrm{mg}, 0.45 \mathrm{mmol})$ and iodomethane $(42 \mu \mathrm{L}$, $0.68 \mathrm{mmol}$ ) were reacted to give 9a as colorless solid (188 $\mathrm{mg}, 91 \%)$. Recrystallization from hexane gave colorless prisms. Mp 128.5-129 ${ }^{\circ} \mathrm{C}$; IR (KBr): 3096, 2948, 2869, 2827, 1718, 1518, 1492, 1459, 1419, 1364, 1306, 1269, 1244, 1160, 1129, 1045, 1009, 883, 786, 730, 647, $579 \mathrm{~cm}^{-1} ;{ }^{1} \mathrm{H}$ NMR (400 MHz, $\left.\mathrm{CDCl}_{3}\right): \delta 1.12(\mathrm{~d}$, $J=7.6 \mathrm{~Hz}, 18 \mathrm{H}), 1.53(\mathrm{~s}, 9 \mathrm{H}), 1.59-1.71(\mathrm{~m}, 3 \mathrm{H}), 2.27(\mathrm{~s}, 6 \mathrm{H}), 2.35(\mathrm{~s}, 3 \mathrm{H}), 3.48(\mathrm{~s}, 2 \mathrm{H})$, $6.95(\mathrm{~s}, 1 \mathrm{H}), 6.98(\mathrm{~d}, J=8.4 \mathrm{~Hz}, 1 \mathrm{H}), 7.17(\mathrm{~d}, J=8.4 \mathrm{~Hz}, 1 \mathrm{H}), 10.36($ br s, $1 \mathrm{H}) ;{ }^{13} \mathrm{C}$ NMR (100 MHz, $\left.\mathrm{CDCl}_{3}\right): \delta 12.71,18.07,28.53,44.30,56.13,78.57,110.76,114.76$, 124.31, 125.16, 125.27, 129.23, 129.85, 140.96, 154.18. Anal. Calcd for $\mathrm{C}_{26} \mathrm{H}_{45} \mathrm{~N}_{3} \mathrm{O}_{2} \mathrm{Si}: \mathrm{C}, 67.92 ; \mathrm{H}, 9.87 ; \mathrm{N}, 9.14$. Found: C, 67.73; H, 10.18; N, 9.16.

\subsubsection{4-(N-tert-Butoxycarbonyl)amino-5-chloro-1-(triisopropylsilyl)gramine (9b).}

According to the general procedure, 8a $(201 \mathrm{mg}, 0.45 \mathrm{mmol})$ and hexachloroethane (160 mg, $0.68 \mathrm{mmol}$ ) were reacted to give $9 \mathbf{b}$ as colorless solid (179 $\mathrm{mg}, 83 \%)$. Recrystallization from diethyl ether-hexane gave colorless prisms. Mp 163-164 ${ }^{\circ} \mathrm{C}$; IR (KBr): 3090, 2948, 2869, 2828, 1724, 1517, 1478, 1416, 1365, 1268, 1251, 1215, 1162, $1041,1017,882,849,786,714,674,647,593,574 \mathrm{~cm}^{-1} ;{ }^{1} \mathrm{H}$ NMR $\left(400 \mathrm{MHz}, \mathrm{CDCl}_{3}\right): \delta$ $1.11(\mathrm{~d}, J=7.5 \mathrm{~Hz}, 18 \mathrm{H}), 1.54(\mathrm{~s}, 9 \mathrm{H}), 1.58-1.71(\mathrm{~m}, 3 \mathrm{H}), 2.27$ (s, 6H), 3.48 (br s, 2H), 
$7.01(\mathrm{~s}, 1 \mathrm{H}), 7.15(\mathrm{~d}, J=8.8 \mathrm{~Hz}, 1 \mathrm{H}), 7.18(\mathrm{~d}, J=8.8 \mathrm{~Hz}, 1 \mathrm{H}), 10.51$ (br s, $1 \mathrm{H}) ;{ }^{13} \mathrm{C}$ NMR (100 MHz, $\left.\mathrm{CDCl}_{3}\right): \delta 12.67,18.00,28.45,44.23,55.72,79.19,111.62,115.21$, 121.45, 123.69, 126.42, 128.52, 130.83, 140.99, 153.77. Anal. Calcd for $\mathrm{C}_{25} \mathrm{H}_{42} \mathrm{ClN}_{3} \mathrm{O}_{2} \mathrm{Si}: \mathrm{C}, 62.54 ; \mathrm{H}, 8.82 ; \mathrm{N}, 8.75$. Found: C, 62.68; H, 9.09; N, 8.70.

\subsubsection{4-(N-tert-Butoxycarbonyl)amino-5-bromo-1-(triisopropylsilyl)gramine (9c).} According to the general procedure, 8a (201 $\mathrm{mg}, 0.45 \mathrm{mmol})$ and 1,2-dibromo-1,1,2,2-tetrafluoroethane $(81 \mu \mathrm{L}, 0.68 \mathrm{mmol})$ were reacted to give 9c as colorless solid (191 mg, 81\%). Recrystallization from diethyl ether-hexane gave

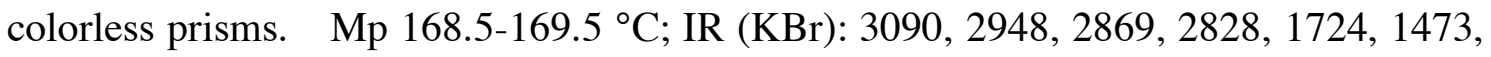
$1415,1365,1268,1251,1215,1161,1146,1040,1015,882,782,586 \mathrm{~cm}^{-1} ;{ }^{1} \mathrm{H}$ NMR $\left(400 \mathrm{MHz}, \mathrm{CDCl}_{3}\right): \delta 1.11(\mathrm{~d}, J=7.5 \mathrm{~Hz}, 18 \mathrm{H}), 1.55(\mathrm{~s}, 9 \mathrm{H}), 1.60-1.70(\mathrm{~m}, 3 \mathrm{H}), 2.26(\mathrm{~s}$, 6H), 3.47 (br s, 2H), 6.99 (s, 1H), 7.12 (d, J=8.8 Hz, 1H), 7.31 (d, J=8.8 Hz, 1H), 10.51 (br s, $1 \mathrm{H}) ;{ }^{13} \mathrm{C} \mathrm{NMR}\left(100 \mathrm{MHz}, \mathrm{CDCl}_{3}\right): \delta 12.66,17.99,28.47,44.25,55.74$, 79.20, 111.25, 112.13, 115.19, 126.47, 126.84, 130.17, 130.70, 141.54, 153.68. Anal. Calcd for $\mathrm{C}_{25} \mathrm{H}_{42} \mathrm{BrN}_{3} \mathrm{O}_{2} \mathrm{Si}$ : C, 57.24; H, 8.07; N, 8.01. Found: C, 56.91; H, 8.22; N, 7.90.

\subsubsection{4-(N-tert-Butoxycarbonyl)amino-5-formyl-1-(triisopropylsilyl)gramine (9d).}

According to the general procedure, 8a $(201 \mathrm{mg}, 0.45 \mathrm{mmol})$ and $N, N$-dimethylformamide (52 $\mu \mathrm{L}, 0.68 \mathrm{mmol}$ ) were reacted to give $9 \mathbf{d}$ as colorless solid (175 mg, 82\%). Recrystallization from diethyl ether-hexane gave colorless prisms. Mp 158-159 ${ }^{\circ} \mathrm{C}$; IR (KBr): 3074, 2948, 2868, 2777, 1723, 1681, 1613, 1575, 1474 , 1422, 1314, 1253, 1160, 1045, 1015, 884, 797, 654, 580, $572 \mathrm{~cm}^{-1} ;{ }^{1} \mathrm{H}$ NMR (400 MHz, $\left.\mathrm{CDCl}_{3}\right): \delta 1.13(\mathrm{~d}, J=7.6 \mathrm{~Hz}, 18 \mathrm{H}), 1.53(\mathrm{~s}, 9 \mathrm{H}), 1.61-1.73(\mathrm{~m}, 3 \mathrm{H}), 2.33(\mathrm{~s}, 6 \mathrm{H}), 3.55$ (s, 2H), 7.07 (s, 1H), $7.27(\mathrm{~d}, J=8.8 \mathrm{~Hz}, 1 \mathrm{H}), 7.73(\mathrm{~d}, J=8.8 \mathrm{~Hz}, 1 \mathrm{H}), 10.12(\mathrm{~s}, 1 \mathrm{H})$, 
11.38 (br s, $1 \mathrm{H}) ;{ }^{13} \mathrm{C}$ NMR $\left(100 \mathrm{MHz}, \mathrm{CDCl}_{3}\right): \delta$ 12.71, 17.98, 28.37, 44.17, 55.71, 79.94, 110.84, 116.41, 121.59, 121.73, 123.91, 130.66, 136.14, 145.94, 155.77, 190.08 . Anal. Calcd for $\mathrm{C}_{26} \mathrm{H}_{43} \mathrm{~N}_{3} \mathrm{O}_{3} \mathrm{Si}: \mathrm{C}, 65.92 ; \mathrm{H}, 9.15 ; \mathrm{N}, 8.87$. Found: C, 65.83; H, 9.19; N, 8.77 .

\subsection{5.}

4-(N-tert-Butoxycarbonyl)amino-5-[hydroxy(phenyl)methyl]-1-(triisopropylsilyl)gr amine (9e). According to the general procedure, 8a (201 $\mathrm{mg}, 0.45 \mathrm{mmol})$ and benzaldehyde $(69 \mu \mathrm{L}, 0.68 \mathrm{mmol})$ were reacted to give 9e as colorless solid (201 $\mathrm{mg}$, 81\%). Recrystallization from diethyl ether-hexane gave colorless prisms. Mp 167-168 ${ }^{\circ} \mathrm{C}$; IR (KBr): 3449, 3179, 3086, 2949, 2869, 2819, 2773, 1702, 1617, 1523, $1457,1422,1367,1275,1254,1159,1043,1018,882,795,709,584 \mathrm{~cm}^{-1} ;{ }^{1} \mathrm{H}$ NMR (400 MHz, $\left.\mathrm{CDCl}_{3}\right): \delta 1.08(\mathrm{~d}, J=7.5 \mathrm{~Hz}, 9 \mathrm{H}), 1.09$ (d, J=7.5 Hz, 9H), $1.55(\mathrm{~s}, 9 \mathrm{H})$, 1.55-1.66 (m, 3H), 2.32 (s, 6H), 3.13 (d, J=12.7 Hz, 1H), 3.89 (d, J=12.7 Hz, 1H), 5.31 (br s, 1H), 6.20 (d, J=2.2 Hz, 1H), 6.82 (d, J= 8.8 Hz, 1H), 7.00 (s, 1H), 7.14 (d, $J=8.8$ $\mathrm{Hz}, 1 \mathrm{H}), 7.21(\mathrm{t}, J=7.5 \mathrm{~Hz}, 1 \mathrm{H}), 7.31(\mathrm{t}, J=7.5 \mathrm{~Hz}, 2 \mathrm{H}), 7.47(\mathrm{~d}, J=7.5 \mathrm{~Hz}, 2 \mathrm{H}), 10.69$ (br s, $1 \mathrm{H}) ;{ }^{13} \mathrm{C}$ NMR $\left(100 \mathrm{MHz}, \mathrm{CDCl}_{3}\right): \delta 12.68,18.03,18.05,28.50,44.39,56.09$, $70.03,80.00,112.05,115.23,123.85,124.72,125.97,126.34,127.53,129.00,130.30$, 131.19, 141.80, 144.30, 157.48. Anal. Calcd for $\mathrm{C}_{32} \mathrm{H}_{49} \mathrm{~N}_{3} \mathrm{O}_{3} \mathrm{Si}: \mathrm{C}, 69.65 ; \mathrm{H}, 8.95 ; \mathrm{N}$, 7.61. Found: C, 69.45; H, 9.09; N, 7.63.

\subsection{6.}

\section{4-(N-tert-Butoxycarbonyl)amino-5-( $N$-tert-butylcarbamoyl)-1-(triisopropylsilyl)gra}

mine (9f). According to the general procedure, 8a $(223 \mathrm{mg}, 0.50 \mathrm{mmol})$ and tert-butyl isocyanate ( $86 \mu \mathrm{L}, 0.75 \mathrm{mmol})$ were reacted to give $9 \mathbf{f}$ as colorless solid (176 $\mathrm{mg}, 65 \%)$. Recrystallization from dichloromethane-pentane gave colorless powder. Mp 
145-147 ${ }^{\circ} \mathrm{C}$; IR (KBr): 3330, 3087, 2951, 2869, 2824, 2778, 1735, 1703, 1655, 1614, $1534,1458,1419,1364,1314,1249,1165,1043,1020,883,651,585 \mathrm{~cm}^{-1} ;{ }^{1} \mathrm{H}$ NMR $\left(400 \mathrm{MHz}, \mathrm{CDCl}_{3}\right): \delta 1.11(\mathrm{~d}, J=7.6 \mathrm{~Hz}, 18 \mathrm{H}), 1.46(\mathrm{~s}, 9 \mathrm{H}), 1.53(\mathrm{~s}, 9 \mathrm{H}), 1.59-1.72(\mathrm{~m}$, 3H), 2.27 (s, 6H), 3.48 (br s, 2H), $6.86($ br s, $1 \mathrm{H}), 7.02(\mathrm{~s}, 1 \mathrm{H}), 7.25(\mathrm{~d}, J=8.6 \mathrm{~Hz}, 1 \mathrm{H})$, $7.51(\mathrm{~d}, J=8.6 \mathrm{~Hz}, 1 \mathrm{H}), 10.65$ (br s, $1 \mathrm{H}) ;{ }^{13} \mathrm{C} \mathrm{NMR}\left(100 \mathrm{MHz}, \mathrm{CDCl}_{3}\right): \delta 12.69,18.01$, $28.67,28.87,44.35,50.80,55.89,79.48,111.03,115.67,123.86,124.90,124.95$, 128.49, 130.75, 143.43, 155.56, 168.01. Anal. Calcd for $\mathrm{C}_{30} \mathrm{H}_{52} \mathrm{~N}_{4} \mathrm{O}_{3} \mathrm{Si}: \mathrm{C}, 66.13 ; \mathrm{H}$, 9.62; N, 10.28. Found: C, 65.92; H, 9.38; N, 10.13.

\subsection{7.}

\section{4-(N-tert-Butoxycarbonyl)amino-5-(N,N-diethylcarbamoyl)-1-(triisopropylsilyl)gra}

mine (9g). According to the general procedure, 8a (201 $\mathrm{mg}, 0.45 \mathrm{mmol})$ and diethylcarbamoyl chloride $(86 \mu \mathrm{L}, 0.68 \mathrm{mmol})$ were reacted to give $\mathbf{9 g}$ as colorless solid (174 mg, 71\%). Recrystallization from diethyl ether-hexane gave colorless powder. Mp 133-134 ${ }^{\circ} \mathrm{C}$; IR (KBr): 3092, 2948, 2869, 2821, 2775, 1724, 1635, 1546, 1458, $1419,1364,1313,1288,1252,1174,1102,1043,1013,882,787,655,583 \mathrm{~cm}^{-1} ;{ }^{1} \mathrm{H}$ $\operatorname{NMR}\left(400 \mathrm{MHz}, \mathrm{CDCl}_{3}\right): \delta 1.11(\mathrm{~d}, J=7.5 \mathrm{~Hz}, 18 \mathrm{H}), 1.18(\mathrm{t}, J=7.1 \mathrm{~Hz}, 3 \mathrm{H}), 1.24(\mathrm{t}, J=$ $7.1 \mathrm{~Hz}, 3 \mathrm{H}), 1.48$ (s, 9H), 1.59-1.71 (m, 3H), 2.26 (s, 6H), 3.14 (br d, J= 11.7 Hz, 1H), 3.19-3.36 (m, 2H), 3.57-3.72 (m, 1H), 3.72-3.87 (m, 2H), $6.98(\mathrm{~s}, 1 \mathrm{H}), 6.99$ (d, J= 8.5 $\mathrm{Hz}, 1 \mathrm{H}), 7.14$ (d, J=8.5 Hz, 1H), 10.80 (br s, $1 \mathrm{H}) ;{ }^{13} \mathrm{C}$ NMR (100 MHz, $\mathrm{CDCl}_{3}$ ): $\delta$ $12.19,12.71,13.59,18.03,28.55,38.09,43.00,44.31,55.86,78.48,109.34,115.76$, $121.55,122.78,124.78,129.51,130.15,142.72,154.29,171.43$. Anal. Calcd for $\mathrm{C}_{30} \mathrm{H}_{52} \mathrm{~N}_{4} \mathrm{O}_{3}$ Si: C, 66.13; H, 9.62; N, 10.28. Found: C, 65.96; H, 9.96; N, 10.28.

\subsection{8.}


(10a). According to the general procedure, $8 \mathbf{b}(476 \mathrm{mg}, 1.0 \mathrm{mmol})$ and iodomethane (93 $\mu \mathrm{L}, 1.5 \mathrm{mmol}$ ) were reacted to give 10a as colorless solid (292 mg, 60\%). Mp 96-98 ${ }^{\circ} \mathrm{C}$; IR (KBr): 3134, 2949, 2868, 2820, 2776, 1728, 1626, 1558, 1456, 1427, 1365 , 1249, 1216, 1171, 1130, 1115, 1048, 1016, 883, 691, 652, $585 \mathrm{~cm}^{-1} ;{ }^{1} \mathrm{H}$ NMR (400 MHz, $\left.\mathrm{CDCl}_{3}\right): \delta 1.13(\mathrm{~d}, J=7.6 \mathrm{~Hz}, 18 \mathrm{H}), 1.53(\mathrm{~s}, 9 \mathrm{H}), 1.60-1.70(\mathrm{~m}, 3 \mathrm{H}), 2.18(\mathrm{~s}, 3 \mathrm{H}), 2.26$ (s, 6H), 3.45 (br s, 2H), 3.81 (s, 3H), $6.78(\mathrm{~s}, 1 \mathrm{H}), 6.85(\mathrm{~s}, 1 \mathrm{H}), 10.42$ (br s, $1 \mathrm{H}) ;{ }^{13} \mathrm{C}$ NMR (100 MHz, $\left.\mathrm{CDCl}_{3}\right): \delta 12.74,18.12,28.52,44.29,56.05,56.10,78.60,94.00$, 114.86, 115.78, 119.26, 127.96, 129.80, 140.42, 154.28, 155.23. Anal. Calcd for $\mathrm{C}_{27} \mathrm{H}_{47} \mathrm{~N}_{3} \mathrm{O}_{3} \mathrm{Si}: \mathrm{C}, 66.21 ; \mathrm{H}, 9.67 ; \mathrm{N}, 8.58$. Found: C, 66.40; H, 10.07; N, 8.61.

\subsection{9.}

\section{4-(N-tert-Butoxycarbonyl)amino-5-chloro-6-methoxy-1-(triisopropylsilyl)gramine}

(10b). According to the general procedure, $8 \mathbf{b}(476 \mathrm{mg}, 1.0 \mathrm{mmol})$ and hexachloroethane (355 $\mathrm{mg}, 1.5 \mathrm{mmol}$ ) were reacted to give $\mathbf{1 0 b}$ as colorless solid (299 $\mathrm{mg}, 59 \%$ ). Recrystallization from diethyl ether-hexane gave colorless prisms. Mp 150-151 ${ }^{\circ} \mathrm{C}$; IR (KBr): 3170, 2948, 2868, 2821, 2776, 1732, 1618, 1559, 1522, 1470, 1427, 1365, 1244, 1213, 1163, 1047, 1022, 884, 691, $651 \mathrm{~cm}^{-1} ;{ }^{1} \mathrm{H}$ NMR (400 MHz, $\left.\mathrm{CDCl}_{3}\right): \delta 1.13(\mathrm{~d}, J=7.6 \mathrm{~Hz}, 18 \mathrm{H}), 1.54(\mathrm{~s}, 9 \mathrm{H}), 1.58-1.67(\mathrm{~m}, 3 \mathrm{H}), 2.26(\mathrm{~s}, 6 \mathrm{H}), 3.45$ (br s, 2H), 3.87 (s, 3H), 6.89 (s, 1H), 6.91 (s, 1H), 10.56 (br s, 1H); ${ }^{13} \mathrm{C}$ NMR (100 MHz, $\left.\mathrm{CDCl}_{3}\right): \delta 12.70,18.05,28.45,44.22,55.70,56.95,79.21,95.56,113.09,115.16$, 120.30, 129.05, 129.56, 140.35, 152.09, 153.61. Anal. Calcd for $\mathrm{C}_{26} \mathrm{H}_{44} \mathrm{ClN}_{3} \mathrm{O}_{3} \mathrm{Si}: \mathrm{C}$, $61.21 ; \mathrm{H}, 8.69 ; \mathrm{N}, 8.24$. Found: C, 61.25; H, 8.82; N, 8.14.

\subsubsection{0.}

4-(N-tert-Butoxycarbonyl)amino-5-bromo-6-methoxy-1-(triisopropylsilyl)gramine (10c). According to the general procedure, $8 \mathbf{b}(476 \mathrm{mg}, 1.0 \mathrm{mmol})$ and 
1,2-dibromo-1,1,2,2-tetrafluoroethane $(178 \mu \mathrm{L}, 1.5 \mathrm{mmol})$ were reacted to give $10 \mathrm{c}$ as colorless solid (311 mg, 56\%). Recrystallization from diethyl ether-hexane gave

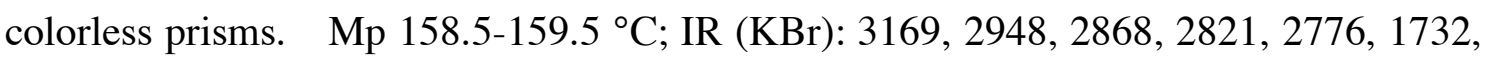
$1612,1559,1517,1467,1425,1365,1244,1212,1163,1019,883,691,650 \mathrm{~cm}^{-1} ;{ }^{1} \mathrm{H}$ $\operatorname{NMR}\left(400 \mathrm{MHz}, \mathrm{CDCl}_{3}\right): \delta 1.13(\mathrm{~d}, J=7.6 \mathrm{~Hz}, 18 \mathrm{H}), 1.54(\mathrm{~s}, 9 \mathrm{H}), 1.58-1.67(\mathrm{~m}, 3 \mathrm{H})$, $2.26(\mathrm{~s}, 6 \mathrm{H}), 3.48$ (br s, 2H), $3.87(\mathrm{~s}, 3 \mathrm{H}), 6.84(\mathrm{~s}, 1 \mathrm{H}), 6.90(\mathrm{~s}, 1 \mathrm{H}), 10.58(\mathrm{br} \mathrm{s}, 1 \mathrm{H})$; ${ }^{13} \mathrm{C} \mathrm{NMR}\left(100 \mathrm{MHz}, \mathrm{CDCl}_{3}\right): \delta 12.69,18.05,28.47,44.24,55.72,57.00,79.19,95.48$, 104.12, 115.09, 120.88, 129.05, 131.16, 141.25, 152.78, 153.53. Anal. Calcd for $\mathrm{C}_{26} \mathrm{H}_{44} \mathrm{BrN}_{3} \mathrm{O}_{3} \mathrm{Si}: \mathrm{C}, 56.30 ; \mathrm{H}, 8.00 ; \mathrm{N}, 7.58$. Found: C, 56.23; H, 8.08; N, 7.40.

\subsubsection{1.}

\section{4-(N-tert-Butoxycarbonyl)amino-5-formyl-6-methoxy-1-(triisopropylsilyl)gramine}

(10d). According to the general procedure, $8 \mathbf{b}(476 \mathrm{mg}, 1.0 \mathrm{mmol})$ and $N, N$-dimethylformamide $(116 \mu \mathrm{L}, 1.5 \mathrm{mmol})$ were reacted to give $\mathbf{1 0 d}$ as colorless solid (247 mg, 49\%). $\quad \mathrm{Mp} \mathrm{103-105}{ }^{\circ} \mathrm{C}$; IR (KBr): 3102, 2949, 2868, 2823, 2775, 1727, 1687, 1621, 1556, 1468, 1424, 1366, 1336, 1246, 1167, 1048, 1012, 884, 692, 650, $581 \mathrm{~cm}^{-1}$; ${ }^{1} \mathrm{H}$ NMR (400 MHz, $\left.\mathrm{CDCl}_{3}\right): \delta 1.14(\mathrm{~d}, J=7.6 \mathrm{~Hz}, 18 \mathrm{H}), 1.52(\mathrm{~s}, 9 \mathrm{H}), 1.58-1.67(\mathrm{~m}$, 3H), 2.30 (s, 6H), 3.48 (br s, 2H), 3.86 (s, 3H), 6.74 (s, 1H), 6.92 (s, 1H), 10.29 (s, 1H), 11.32 (br s, $1 \mathrm{H}) ;{ }^{13} \mathrm{C}$ NMR $\left(100 \mathrm{MHz}, \mathrm{CDCl}_{3}\right): \delta$ 12.71, 18.03, 28.43, 44.12, 55.64, $56.38,79.74,93.61,113.40,116.48,118.48,129.25,134.87,145.82,154.59,157.76$, 189.48. Anal. Calcd for $\mathrm{C}_{27} \mathrm{H}_{45} \mathrm{~N}_{3} \mathrm{O}_{4} \mathrm{Si}: \mathrm{C}, 64.38 ; \mathrm{H}, 9.00 ; \mathrm{N}, 8.34$. Found: C, 64.44; H, 9.31; N, 8.33.

\subsubsection{2.}

4-(N-tert-Butoxycarbonyl)amino-5-[hydroxy(phenyl)methyl]-6-methoxy-1-(triisopr opylsilyl)gramine (10e). According to the general procedure, $8 \mathbf{b}$ (476 mg, $1.0 \mathrm{mmol})$ 
and benzaldehyde $(152 \mu \mathrm{L}, 1.5 \mathrm{mmol})$ were reacted to give $\mathbf{1 0 e}$ as colorless solid (304 mg, 52\%). $\operatorname{Mp~111-113~}{ }^{\circ} \mathrm{C}$; IR (KBr): 3386, 2949, 2868, 2821, 2776, 1702, 1622, $1557,1467,1426,1367,1277,1253,1169,1048,1016,883,694,652,593 \mathrm{~cm}^{-1} ;{ }^{1} \mathrm{H}$ NMR (400 MHz, $\left.\mathrm{CDCl}_{3}\right): \delta 1.13(\mathrm{~d}, J=7.5 \mathrm{~Hz}, 18 \mathrm{H}), 1.49(\mathrm{~s}, 9 \mathrm{H}), 1.55-1.68(\mathrm{~m}, 3 \mathrm{H})$, $2.27(\mathrm{~s}, 6 \mathrm{H}), 3.26(\mathrm{~d}, J=12.5 \mathrm{~Hz}, 1 \mathrm{H}), 3.47$ (s, 3H), 3.64 (d, J=12.5 Hz, 1H), 4.99 (br s, $1 \mathrm{H}), 6.21(\mathrm{~d}, J=6.5 \mathrm{~Hz}, 1 \mathrm{H}), 6.78(\mathrm{~s}, 1 \mathrm{H}), 6.89(\mathrm{~s}, 1 \mathrm{H}), 7.10-7.16(\mathrm{~m}, 1 \mathrm{H}), 7.20-7.26(\mathrm{~m}$, 2H), 7.32-7.37 (m, 2H), 10.58 (br s, $1 \mathrm{H}) ;{ }^{13} \mathrm{C} \mathrm{NMR} \mathrm{(100} \mathrm{MHz,} \mathrm{CDCl}_{3}$ ): $\delta$ 12.71, 18.07, $28.47,44.27,55.96,56.26,69.22,79.64,96.41,115.38,119.45,121.55,125.06,125.22$, 126.98, 128.70, 130.01, 142.14, 145.46, 155.73, 156.60. Anal. Calcd for $\mathrm{C}_{33} \mathrm{H}_{51} \mathrm{~N}_{3} \mathrm{O}_{4} \mathrm{Si}: \mathrm{C}, 68.12 ; \mathrm{H}, 8.83 ; \mathrm{N}, 7.22$. Found: C, 68.07; H, 9.07; N, 7.15.

\subsubsection{3.}

\section{4-(N-tert-Butoxycarbonyl)amino-5-(N-tert-butylcarbamoyl)-6-methoxy-1-(triisopro}

pylsilyl)gramine (10f). According to the general procedure, $8 \mathbf{b}$ (476 mg, $1.0 \mathrm{mmol}$ ) and tert-butyl isocyanate $(171 \mu \mathrm{L}, 1.5 \mathrm{mmol})$ were reacted to give $\mathbf{1 0 f}$ as colorless solid (235 mg, 41\%). Recrystallization from diethyl ether-hexane gave colorless powder. $\operatorname{Mp~156-158~}{ }^{\circ} \mathrm{C}$; IR (KBr): 3360, 3187, 2952, 2868, 2821, 2774, 1715, 1649, 1624, $1543,1459,1365,1310,1251,1207,1161,1049,1027,1015,882,689,654,571,524$ $\mathrm{cm}^{-1} ;{ }^{1} \mathrm{H}$ NMR $\left(400 \mathrm{MHz}, \mathrm{CDCl}_{3}\right): \delta 1.12(\mathrm{~d}, J=7.5 \mathrm{~Hz}, 18 \mathrm{H}), 1.46(\mathrm{~s}, 9 \mathrm{H}), 1.52(\mathrm{~s}, 9 \mathrm{H})$, 1.55-1.67 (m, 3H), $2.20(\mathrm{~s}, 6 \mathrm{H}), 3.41(\mathrm{~s}, 2 \mathrm{H}), 3.81(\mathrm{~s}, 3 \mathrm{H}), 6.81(\mathrm{~s}, 1 \mathrm{H}), 6.84(\mathrm{br} \mathrm{s}, 1 \mathrm{H})$, $6.88(\mathrm{~s}, 1 \mathrm{H}), 10.11$ (br s, $1 \mathrm{H}) ;{ }^{13} \mathrm{C} \mathrm{NMR}\left(100 \mathrm{MHz}, \mathrm{CDCl}_{3}\right): \delta 12.69,18.05,28.66$, $28.70,44.40,51.05,56.66,79.30,95.39,115.59,119.84,119.86,128.34,129.03$, 137.80, 142.45, 153.85, 156.38, 166.21. Anal. Calcd for $\mathrm{C}_{31} \mathrm{H}_{54} \mathrm{~N}_{4} \mathrm{O}_{4} \mathrm{Si}: \mathrm{C}, 64.77 ; \mathrm{H}$, 9.47; N, 9.75. Found: C, 64.73; H, 9.57; N, 9.92.

\subsubsection{4.}




\section{4-(N-tert-Butoxycarbonyl)amino-5-( $N, N$-diethylcarbamoyl)-6-methoxy-1-(triisopro}

pylsilyl)gramine (10g). According to the general procedure, $\mathbf{8 b}(476 \mathrm{mg}, 1.0 \mathrm{mmol})$ and diethylcarbamoyl chloride $(190 \mu \mathrm{L}, 1.5 \mathrm{mmol})$ were reacted to give $\mathbf{1 0 g}$ as colorless solid (213 mg, 37\%). Recrystallization from hexane gave colorless powder. Mp 184-186 ${ }^{\circ} \mathrm{C}$; IR (KBr): 3134, 2948, 2868, 2819, 2775, 1727, 1623, 1557, 1457, 1427 , $1289,1250,1212,1170,1142,1046,1014,883,787,692,651,610,524 \mathrm{~cm}^{-1} ;{ }^{1} \mathrm{H}$ NMR $\left(400 \mathrm{MHz}, \mathrm{CDCl}_{3}\right): \delta 1.13(\mathrm{~d}, J=7.5 \mathrm{~Hz}, 9 \mathrm{H}), 1.13(\mathrm{~d}, J=7.5 \mathrm{~Hz}, 9 \mathrm{H}), 1.17(\mathrm{t}, J=7.3$ $\mathrm{Hz}, 3 \mathrm{H}), 1.22(\mathrm{t}, J=7.1 \mathrm{~Hz}, 3 \mathrm{H}), 1.47(\mathrm{~s}, 9 \mathrm{H}), 1.57-1.68(\mathrm{~m}, 3 \mathrm{H}), 2.21(\mathrm{~s}, 6 \mathrm{H}), 3.02(\mathrm{~d}$, $J=12.5 \mathrm{~Hz}, 1 \mathrm{H}), 3.23-3.39(\mathrm{~m}, 2 \mathrm{H}), 3.49-3.59(\mathrm{~m}, 1 \mathrm{H}), 3.75(\mathrm{~s}, 3 \mathrm{H}), 3.80(\mathrm{~d}, J=12.5$ $\mathrm{Hz}, 1 \mathrm{H}), 3.81-3.90(\mathrm{~m}, 1 \mathrm{H}), 6.71(\mathrm{~s}, 1 \mathrm{H}), 6.86$ (s, 1H), 10.28 (br s, 1H); ${ }^{13} \mathrm{C}$ NMR (100 $\left.\mathrm{MHz}, \mathrm{CDCl}_{3}\right): \delta 12.55,12.72,12.97,18.06,18.09,28.53,37.53,43.00,44.42,55.58$, $55.80,78.54,93.09,115.76,116.66,119.76,128.65,129.52,142.51,152.84,154.60$, 167.34. Anal. Calcd for $\mathrm{C}_{31} \mathrm{H}_{54} \mathrm{~N}_{4} \mathrm{O}_{4} \mathrm{Si}$ : C, 64.77; H, 9.47; N, 9.75. Found: C, 64.63; H, 9.74; N, 9.80.

\section{References and note}

1. Sundberg, R. J. Indoles; Academic Press: London, 1996.

2. (a) Somei, M.; Hasegawa, T.; Kaneko, C. Heterocycles 1983, 20, 1983-1985; (b) Somei, M.; Saida, Y. Heterocycles 1985, 23, 3113-3114; (c) Moyer, M. P.; Shiurba, J. F.; Rapoport, H. J. Org. Chem. 1986, 51, 5106-5110; (d) Beswick, P. J.; Greenwood, C. S.; Mowlem, T. J.; Nechvatal, G.; Widdowson, D. A. Tetrahedron, 1988, 44, 7325-7334; (e) Masters, N. F.; Mathews, N.; Nechvatal, G.; Widdowson, D. A. Tetrahedron, 1989, 45, 5955-5970; (f) Iwao, M.; Kuraishi, T. Heterocycles, 1992, 34, 1031-1038; (g) Iwao, M. Heterocycles, 1993, 36, 29-32; (h) Nakagawa, 
K.; Somei, M. Heterocycles, 1994, 39, 31-34; (i) Griffen, E. J.; Roe, D. G.; Snieckus, V. J. Org. Chem. 1995, 60, 1484-1485; (k) Fukuda, T.; Maeda. R.; Iwao, M. Tetrahedron 1999, 55, 9151-9162; (1) Hartung, C. G.; Fecher, A.; Chapell, B.; Snieckus, V. Org. Lett. 2003, 5, 1899-1902.

3. Iwao, M.; Motoi, O. Tetrahedron Lett. 1995, 36, 5929-5932.

4. (a) Iwao, M.; Ishibashi, F. Tetrahedron 1997, 53, 51-58; (b) Shinohara, H.; Fukuda, T.; Iwao, M. Tetrahedron 1999, 55, 10989-11000.

5. (a) Iwao, M.; Motoi, O.; Fukuda, T.; Ishibashi, F. Tetrahedron 1998, 54, 8999-9010; (b) Moro-oka, Y.; Fukuda, T.; Iwao, M. Tetrahedron Lett. 1999, 40, 1713-1716; (c) Moro-oka, Y.; Iwakiri, S.; Fukuda, T.; Iwao, M. Tetrahedron Lett. 2000, 41, $5225-5228$.

6. (a) Meseguer, B.; Alonso-Díaz, D.; Griebenow, N.; Herget, T.; Waldmann, H. Angew. Chem. Int. Ed. 1999, 38, 2902-2906; (b) Meseguer, B.; Alonso-Díaz, D.; Griebenow, N.; Herget, T.; Waldmann, H. Chem. Eur. J. 2000, 6, 3943-3957.

7. Hayakawa, Y.; Singh, M.; Shibata, N.; Takeuchi, Y.; Kirk, K. L. J. Fluorine Chem. 1999, 97, 161-164.

8. (a) Nettekoven, M.; Psiorz, M.; Waldmann, H. Tetrahedron Lett. 1995, 36, 1425-1428; (b) Hurt, C. R.; Lin, R.; Rapoport, H. J. Org. Chem. 1999, 64, 225-233; (c) Pérez-Serrano, L.; Casarrubios, L.; Domínguez, G.; Freire, G.; Pérez-Castells, J. Tetrahedron 2002, 58, 5407-5415.

9. Chauder, B.; Larkin, A.; Snieckus, V. Org. Lett. 2002, 4, 815-817.

10. (a) Mills, R. J.; Taylor, N. J.; Snieckus, V. J. Org. Chem. 1989, 54, 4372-4385; (b) Snieckus, V. Chem. Rev. 1990, 90, 879-933; (c) Hong, H.; Comins, D. L. J. Org. Chem. 1996, 61, 391-392.

11. (a) Muchowski, J. M.; Venuti, M. C. J. Org. Chem. 1980, 45, 4798-4891; (b) Reed, J. N.; Rotchford, J.; Strickland, D. Tetrahedron Lett. 1988, 29, 5725-5728; (c) Cho, 
I.-S.; Gong, L.; Muchowski, J. M. J. Org. Chem. 1991, 56, 7288-7291; (d) Stanetty, P.; Koller, H.; Mihovilovic, M. J. Org. Chem. 1992, 57, 6833-6837.

12. Somei, M.; Tsuchiya, M. Chem. Pharm. Bull. 1981, 29, 3145-3157.

13. (a) Nishiyama, K.; Tanaka, N. J. Chem. Soc., Chem. Commun. 1983, 1322-1323;

(b) Tsuge, O.; Kanemasa, S.; Matsuda, K. Chem. Lett. 1983, 1131-1134.

14. It has been reported that $N$-tert-butoxycarbonyl-3-methoxyaniline is not lithiated cleanly or efficiently: see, ref. $11 b$, c. 

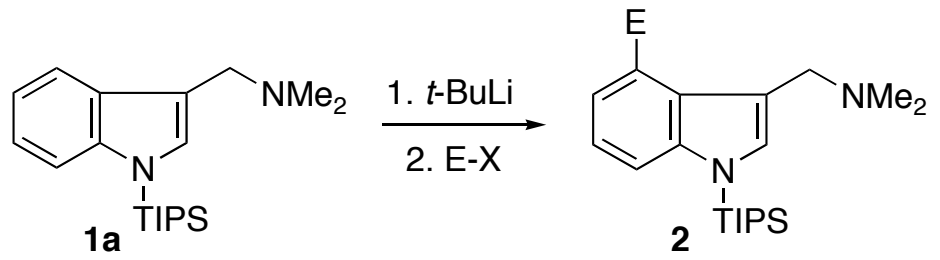

Scheme 1. Directed lithiation of 1-(triisopropylsilyl)gramine (1a)

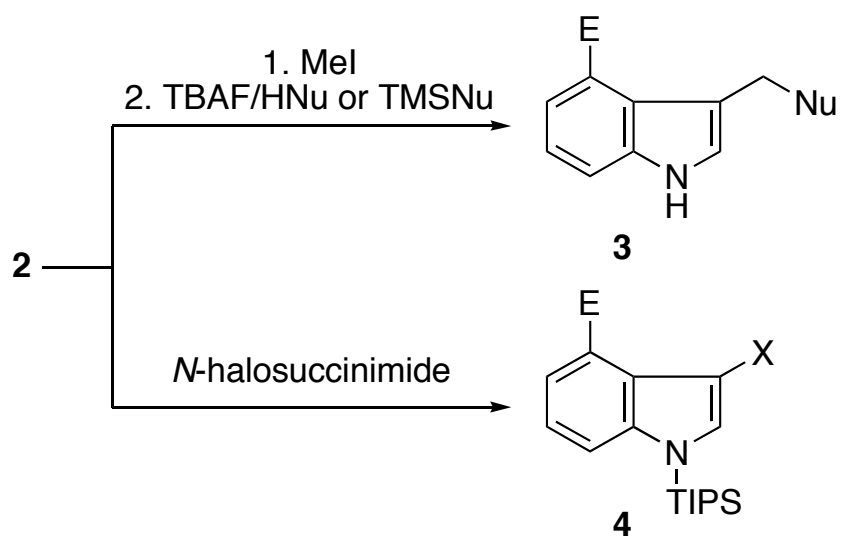

Scheme 2. Functionalization at C-3 (side chain or ring) of gramines 2 


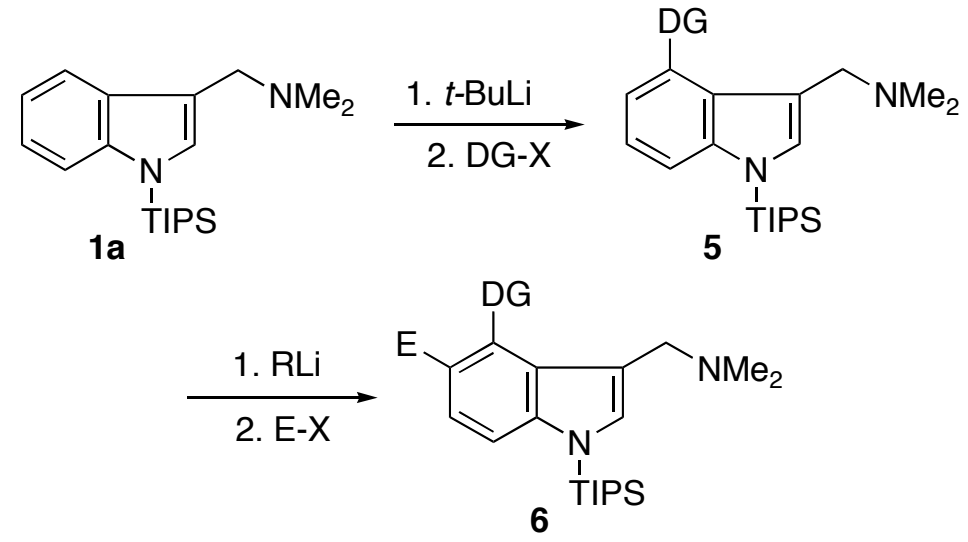

Scheme 3. Iterative directed lithiation of 1a to produce 3,4,5-trisubstituted indoles 6

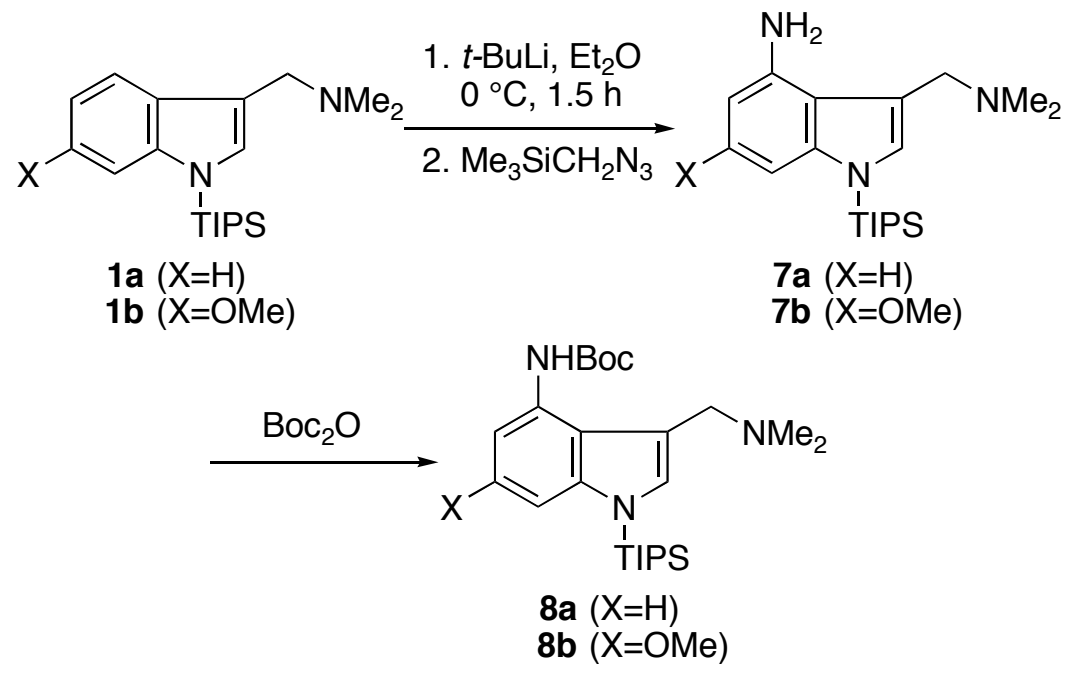

Scheme 4. Synthesis of 4-(N-tert-butoxycarbonyl)amino-1-(triisopropylsilyl)gramines 8 
Table.

Directed lithiation-functionalization at

C-5 of

4-(N-tert-butoxycarbonyl)amino-1-(triisopropylsilyl)gramines 8

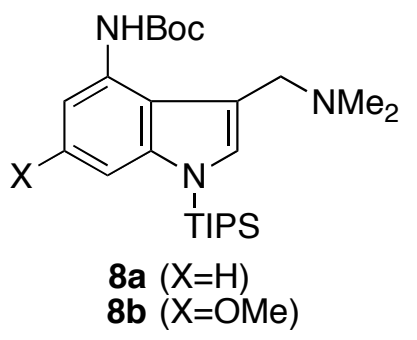

$8 \mathbf{a}(X=\mathrm{H})$
$\mathbf{8 b}(\mathrm{X}=\mathrm{OMe})$

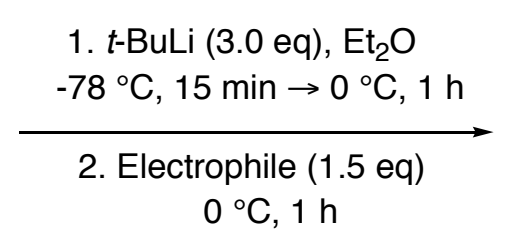

$0^{\circ} \mathrm{C}, 1 \mathrm{~h}$<smiles></smiles>

$9(\mathrm{X}=\mathrm{H})$

$10(\mathrm{X}=\mathrm{OMe})$

\begin{tabular}{|c|c|c|c|c|c|}
\hline Entry & Substrate & Electrophile & $\mathrm{E}$ & Product & Yield $(\%)^{\mathrm{a}}$ \\
\hline 1 & $8 \mathbf{a}$ & MeI & $\mathrm{Me}$ & 9a & 91 \\
\hline 2 & $8 \mathbf{a}$ & $\mathrm{Cl}_{3} \mathrm{CCCl}_{3}$ & $\mathrm{Cl}$ & $9 b$ & 83 \\
\hline 3 & $8 \mathbf{a}$ & $\mathrm{BrF}_{2} \mathrm{CCBrF}_{2}$ & $\mathrm{Br}$ & $9 c$ & 86 \\
\hline 4 & $8 \mathbf{a}$ & DMF & $\mathrm{CHO}$ & 9d & 82 \\
\hline 5 & $8 \mathbf{a}$ & $\mathrm{PhCHO}$ & $\mathrm{CH}(\mathrm{OH}) \mathrm{Ph}$ & $9 e$ & 81 \\
\hline 6 & $\mathbf{8 a}$ & $t$-BuNCO & $\mathrm{CONH}(t-\mathrm{Bu})$ & $9 f$ & 65 \\
\hline 7 & $8 \mathbf{a}$ & $\mathrm{Et}_{2} \mathrm{NCOCl}$ & $\mathrm{CONEt}_{2}$ & $9 g$ & 71 \\
\hline 8 & $8 \mathbf{b}$ & MeI & $\mathrm{Me}$ & $10 \mathrm{a}$ & 60 \\
\hline 9 & $8 b$ & $\mathrm{Cl}_{3} \mathrm{CCCl}_{3}$ & $\mathrm{Cl}$ & $10 \mathrm{~b}$ & 59 \\
\hline 10 & $8 b$ & $\mathrm{BrF}_{2} \mathrm{CCBrF}_{2}$ & $\mathrm{Br}$ & $10 \mathrm{c}$ & 56 \\
\hline 11 & $8 \mathbf{b}$ & $\mathrm{DMF}$ & $\mathrm{CHO}$ & 10d & 49 \\
\hline 12 & $8 \mathbf{b}$ & $\mathrm{PhCHO}$ & $\mathrm{CH}(\mathrm{OH}) \mathrm{Ph}$ & $10 \mathrm{e}$ & 52 \\
\hline 13 & $8 \mathbf{b}$ & $t$-BuNCO & $\mathrm{CONH}(t-\mathrm{Bu})$ & $10 f$ & 41 \\
\hline 14 & $8 \mathbf{b}$ & $\mathrm{Et}_{2} \mathrm{NCOCl}$ & $\mathrm{CONEt}_{2}$ & $10 \mathrm{~g}$ & 37 \\
\hline
\end{tabular}

${ }^{\mathrm{a}}$ Isolated yield. 


\section{Graphical Abstract}

Synthesis of 3,4,5-trisubstituted indoles via iterative directed lithiation of 1-(triisopropylsilyl)gramines

Tsutomu Fukuda, Hiroko Akashima and Masatomo Iwao*

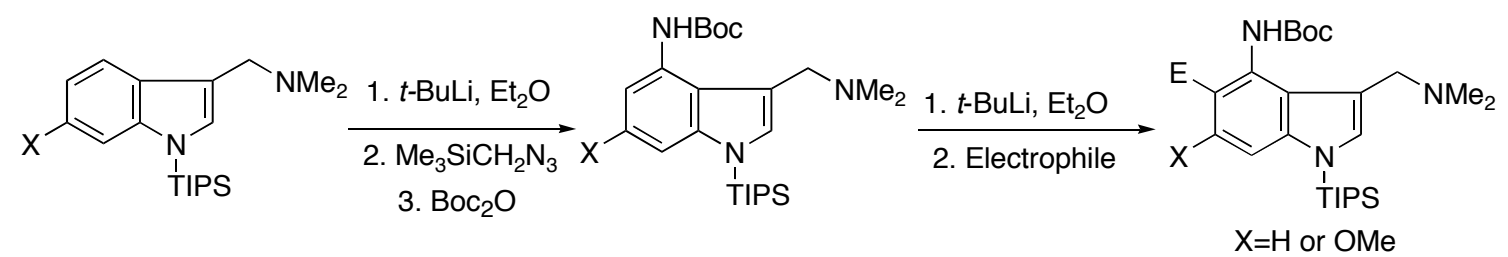

